\title{
ELASTIC-PLASTIC STRESSES IN A THIN ROTATING DISK WITH SHAFTHAVING DENSITY VARIATION PARAMETER UNDER STEADY-STATE TEMPERATURE
}

\author{
Pankaj Thakur ${ }^{1}$, Satya Bir Singh ${ }^{2}$ and Jatinder Kaur ${ }^{3}$ \\ ${ }^{1}$ Department of Mathematics, IEC University Baddi, \\ Solan, Himachal Pradesh 174103, India \\ E-mails: pankaj_thakur15@yahoo.co.in,dr_pankajthakur@yahoo.com \\ ${ }^{2}$ Department of Mathematics, Punjabi University Patiala, Punjab 147002, India \\ ${ }^{3}$ Department of Applied Science, Rayat Institute of Engineering \& Information Technology, \\ Ropar, Punjab, India
}

(Received June 8, 2013)

\begin{abstract}
Steady thermal stresses in a rotating disc with shaft having density variation parameter subjected to thermal load have been derived by using Seth's transition theory. Neither the yields criterion nor the associated flow rule is assumed here. Results are depicted graphically. It has been seen that compressible material required higher percentage increased angular speed to become fully-plastic as compare to rotating disc made of incompressible material. Circumferential stresses are maximal at the outer surface of the rotating disc. With the introduction of thermal effect it decreases the value of radial and circumferential stresses at inner and outer surface for fully-plastic state.
\end{abstract}

Key words: Stresses, displacement, rotating disc, angular speed, shaft, temperature, density.

\section{INTRODUCTION}

Disc plays an important role in machine design. Stress analysis of rotating discs has an important role in engineering design. Rotating discs are the most critical part of rotors, turbines motor, compressors, high speed gears, flywheel, sink fits, turbo jet engines and computer's disc drive etc.The problem of thin rotating flat discs made of isotropic material has been studied extensively [1-3]. CHAKRABARTY [1] and HEYMAN [2] solved the problem for the plastic state by utilizing the solution in the elastic state and consider the plastic range with the help of Tresca's yield condition. Further, to obtain the elastic-plastic stresses, these authors matched the elastic and plastic stresses at the same radius $r=c$ of the disc. Perfectly elasticity and ideal plasticity are two extreme properties of the material and the use of ad-hoc rule like yield condition amounts to divide the two extreme properties by a sharp line, which is not physically possible. Seth's transition theory[4] does not required any assumptions like an yield criterion, incompressibility condition, associated flow rule and thus poses and solves a more general problem from which cases pertaining to the above assumptions can be worked out. This theory [4] utilizes the concept of generalized strain measure and asymptotic solution at critical points or turning points of the 
differential equations defining the deformed field and has been successfully applied to a large number of problems [4-19].SETH [5] has defined the generalized principal strain measure as:

$e_{i i}=\int_{0}^{A} e_{i i}^{A}\left[1-2 e_{i i}^{A}\right]^{\frac{n}{2}-1} d \hat{e}_{i i}^{A}=\frac{1}{n}\left[1-\left(1-2 \hat{e}_{i i}\right)\right],(i=1,2,3)$

where $n$ is the measure and $e_{i i}$ is the almansi finite strain components. For $n=-2,-1,0,1,2$ it gives Cauchy, Green Hencky, Swainger and Almansi measures, respectively.

In this research paper we discusselastic-plastic transitional stresses in a thin rotating disk with shaft having density variation parameter under steady state temperature by using Seth's transition theory. The density of disc is assumed to vary along the radius in the form:

$\rho=\rho_{0}(r / b)^{-m}$

where $\rho_{0}$ is the constant density at $r=b$ and mis the density variation parameter. Result obtained have been numerically and depicted graphically.

\section{MATHEMATICAL MODEL}

Consider a thin disc of isotropic and homogeneous material having variable density with central bore of inner radius $a$ and external radius $b$. The annular disc is mounted on a rigid shaft. The disc is rotating with angular speed $\omega$ of gradually increasing magnitude about an axis perpendicular to its plane and passed through the center as shown in Fig. 1. The thickness of disc is assumed to be constant and sufficiently small so that it is effectively in a state of plane stress, that is, the axial stress $T_{z z}$ is zero. We assume that steady state temperature $\Theta_{0}$ is applied on the internal surface of the disc.

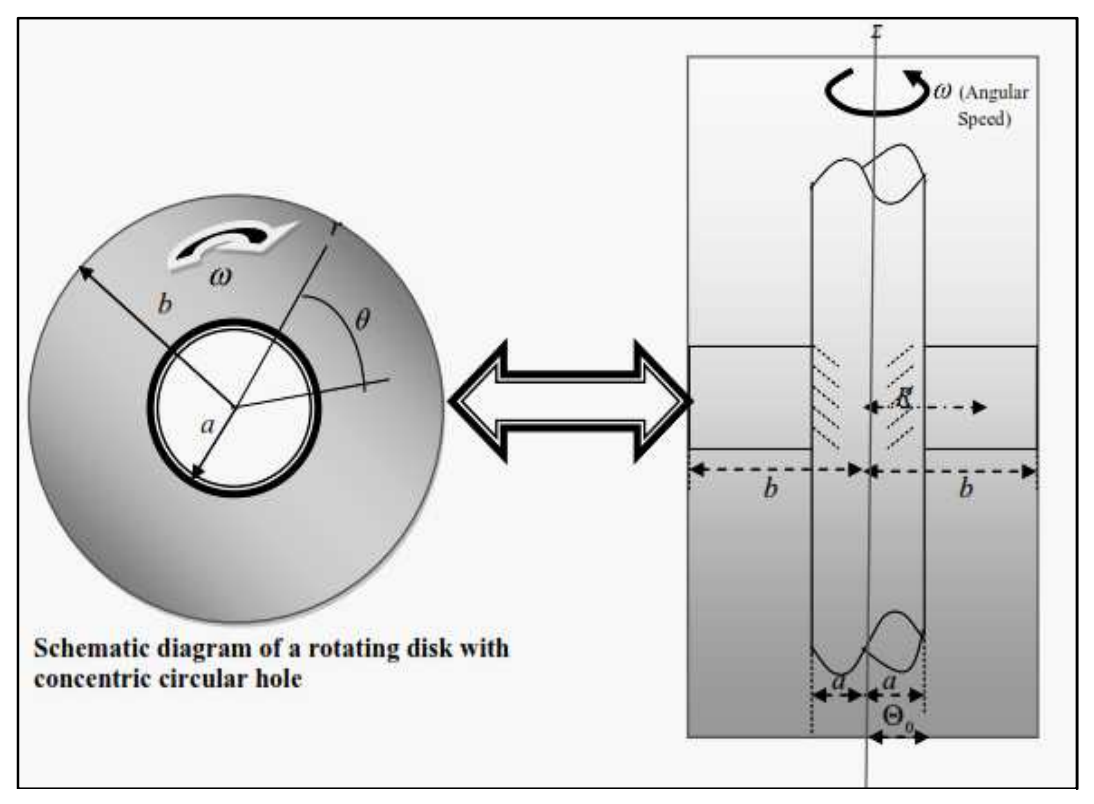

Figure 1. Geometry of Rotating Disc.

\section{Boundary conditions}

The disk considered in the present study having variable density and subjected to a thermal load. The inner surface of the disk is assumed to be fixed to a shaft. The outer surface of the disk is free from mechanical load. Thus, the boundary conditions of the problem are given by:

(i) $\quad r=a u=0$

(ii) $\quad r=b, T_{r r}=0$

where $u$ and $T_{r r}$ denote displacement and stress along the radial direction.

\section{Formulation of the Problem}

Displacement components in cylindrical polar coordinates $(r, \theta, z)$, as: 
$u=r(1-\beta), v=0, w=d z$

where $\beta$ is function of $r=\sqrt{x^{2}+y^{2}}$ only and $d$ is a constant.

The finite strain components are given by Seth [5]:

$e_{r r}^{A} \equiv \frac{\partial u}{\partial r}-\frac{1}{2}\left(\frac{\partial u}{\partial r}\right)^{2}=\frac{1}{2}\left[1-\left(r \beta^{\prime}+\beta\right)^{2}\right], e_{\theta \theta}^{A} \equiv \frac{u}{r}-\frac{u^{2}}{2 r^{2}}=\frac{1}{2}\left[1-\beta^{2}\right]$

$\stackrel{A}{e_{z z}} \equiv \frac{\partial w}{\partial z}-\frac{1}{2}\left(\frac{\partial w}{\partial z}\right)^{2}=\frac{1}{2}\left[1-(1-d)^{2}\right], \stackrel{A}{e_{r \theta}} \stackrel{A}{A} e_{\theta z}=\stackrel{A}{=} e_{z r}=0$

where $\beta^{\prime}=d \beta / d r$ and meaning of superscripts “A”" is Almansi.

Substituting eq.(5)in eq. (1), the generalized components of strain are given by:

$e_{r r}=\frac{1}{n}\left[1-\left(r \beta^{\prime}+\beta\right)^{n}\right], e_{\theta \theta}=\frac{1}{n}\left[1-\beta^{n}\right], e_{z z}=\frac{1}{n}\left[1-(1-d)^{n}\right], e_{r \theta}=e_{\theta z}=e_{z r}=0$

where $\beta^{\prime}=d \beta / d r$.

The stress -strain relations for thermo elastic isotropic material are given by [20]:

$T_{i j}=\lambda \delta_{i j} I_{1}+2 \mu e_{i j}-\xi \Theta \delta_{i j},(i, j=1,2,3)$

where $T_{i j}$ is the stress components, $\lambda$ and $\mu$ are Lame's constants and $I_{1}=e_{k k}$ is the first strain invariant, $\delta_{i j}$ is the Kronecker's delta and $\xi=\alpha(3 \lambda+2 \mu), \alpha$ being the coefficient of thermal expansion and $\Theta$ is the rise of temperature. Further, $\Theta$ has to satisfy

$\nabla^{2} \Theta=0$

Eq. (7) for this problem becomes:

$T_{r r}=\frac{2 \lambda \mu}{\lambda+2 \mu}\left[e_{r r}+e_{\theta \theta}\right]+2 \mu e_{r r}-\frac{2 \mu \xi \Theta}{(\lambda+2 \mu)}, T_{\theta \theta}=\frac{2 \lambda \mu}{\lambda+2 \mu}\left[e_{r r}+e_{\theta \theta}\right]+2 e_{\theta \theta}-\frac{2 \mu \xi \Theta}{(\lambda+2 \mu)}$,

$T_{r \theta}=T_{\theta z}=T_{z r}=T_{z z}=0$

Substituting eq. (5) in eq. (6), the strain components in terms of stresses are obtained as [6]:

$e_{r r}=\frac{\partial u}{\partial r}-\frac{1}{2}\left(\frac{\partial u}{\partial r}\right)^{2}=\frac{1}{2}\left[1-\left(r \beta^{\prime}+\beta\right)^{2}\right]=\frac{1}{E}\left[T_{r r}-\left(\frac{1-C}{2-C}\right) T_{\theta \theta}\right]+\alpha \Theta$,

$e_{\theta \theta}=\frac{u}{r}-\frac{u^{2}}{2 r^{2}}=\frac{1}{2}\left[1-\beta^{2}\right]=\frac{1}{E}\left[T_{\theta \theta}-\left(\frac{1-C}{2-C}\right) T_{r r}\right]+\alpha \Theta$,

$e_{z z}=\frac{\partial w}{\partial z}-\frac{1}{2}\left(\frac{\partial w}{\partial z}\right)^{2}=\frac{1}{2}\left[1-(1-d)^{2}\right]=-\frac{(1-C)}{E(2-C)}\left[T_{r r}-T_{\theta \theta}\right]+\alpha \Theta$,

$e_{r \theta}=e_{\theta z}=e_{z r}=0$.

where $E$ is the Young's modulus and $C$ is compressibility factor of the material in term of Lame's constant, there are given by $E=\mu(3 \lambda+2 \mu) /(\lambda+\mu)$ and $C=2 \mu /(\lambda+2 \mu)$.

Substituting eq. (6) in eq. (9), one get

$T_{r r}=\frac{2 \mu}{n}\left[3-2 C-\beta^{n}\left\{1-C+(2-C)(P+1)^{n}+\frac{n C \xi \Theta}{2 \mu \beta^{n}}\right\}\right.$,

$T_{\theta \theta}=\frac{2 \mu}{n}\left[3-2 C-\beta^{n}\left\{2-C+(1-C)(P+1)^{n}+\frac{n C \xi \Theta}{2 \mu \beta^{n}}\right\}\right.$,

$T_{r \theta}=T_{\theta_{z}}=T_{z r}=T_{z z}=0$.

where $r \beta^{\prime}=\beta P$. 
The equations of equilibrium are all satisfied except:

$\frac{d}{d r}\left(r T_{r r}\right)-T_{\theta \theta}+\rho \omega^{2} r^{2}=0$

where $\rho$ variable density of the material of the rotating disc.

The temperature field satisfying Laplace eq. (8) with boundary condition

$\Theta=\Theta_{0}$ at $r=a$

$\Theta=0$ at $r=b$.

where $\Theta_{0}$ is constant, given by:

$\Theta=\Theta_{0} \frac{\ln (r / b)}{\ln (a / b)}$

Using eqs. (11), (12) and (13), one gets a non- linear differential equation in $\beta$ as:

$(2-C) n \beta^{n+1} P(P+1)^{n-1} \frac{d P}{d \beta}=\left[\frac{n \rho \omega^{2} r^{2}}{2 \mu}+\beta^{n}\left[\begin{array}{l}1-(P+1)^{n} \\ -n P\left\{1-C+(2-C)(P+1)^{n}\right\}\end{array}\right]-\frac{n C \xi \bar{\Theta}_{0}}{2 \mu}\right]$

where $\bar{\Theta}_{0}=\Theta_{0} / \ln (a / b)$ and $r \beta^{\prime}=\beta P$ ( $P$ is function of $\beta$ and $\beta$ is function of $r$ ) and $\beta^{\prime}=d \beta / d r(P$ is function of $\beta$ and $\beta$ is function of $r$ only).

\section{Solution through the Problem}

For finding the plastic stress, the transition function is taken through the principal stress (see Seth's [4, 5] and PANKaJ Thakur [6-21]) at the transition point $P \rightarrow \pm \infty$. The transition function $\tau$ is defined as:

$\tau=\frac{n}{2 \mu}\left[T_{\theta \theta}-C \xi \Theta\right]=\left[(3-2 C)-\beta^{n}\left\{2-C+(1-C)(P+1)^{n}\right\}-\frac{n C \xi \Theta}{\mu}\right]$

Taking the logarithmic differentiation of eq. (15) with respect to $r$, one get:

$\frac{d(\log \tau)}{d r}=-\left(\frac{n \beta^{n} P}{r}\right)\left[\frac{2-C+(1-C)(P+1)^{n-1}\left\{(P+1)+\beta \frac{d P}{d \beta}\right\}}{(3-2 C)-\beta^{n}\left\{2-C+(1-C)(P+1)^{n}\right\}-\frac{n C \xi \Theta}{\mu}}\right]$

Substituting the value $d P / d \beta$ from eq. (14) in eq. (16) and taking the asymptotic value $P \rightarrow \pm \infty$ and integrating, one get:

$\tau=A r^{\nu-1}$

where $v=1-C / 2-C$ and $A$ is a constant of integration can be determined by boundary conditions.

Eqs. (15) and (17) gives:

$T_{\theta \theta}=\left(\frac{2 \mu}{n}\right) A r^{\nu-1}+\frac{C \xi \Theta_{0} \ln (r / b)}{\ln (a / b)}$

Substituting eq. (18) in eq. (12) then using eq. (2) and integrating, one get:

$T_{r r}=\left(\frac{2 \mu}{n \nu}\right) A r^{\nu-1}-\frac{\rho_{0} \omega^{2} b^{m} r^{2-m}}{(3-k)}+\frac{B}{r}+\frac{C \xi \Theta_{0} \ln (r / b)}{\ln (a / b)}-\frac{C \xi \Theta_{0}}{\ln (a / b)}$

where $B$ is a constant of integrationcan be determined by boundary conditions. 
Substituting eqs. (18) and (19) in second equation of eq. (10), one get:

$\beta=\sqrt{1-\frac{2 v}{E}\left[\frac{\rho_{0} \omega^{2} b^{m} r^{2-m}}{3-m}-\frac{B_{1}}{r}+\frac{\alpha E \Theta_{0}(2-C)}{\ln (a / b)}+\frac{2(2-C) \alpha E \Theta_{0} \ln (r / b)}{\ln (a / b)}\right]}$

where $C \xi=\alpha E(2-C)$.

Substituting eq. (20) in eq. (4), one get:

$u=r-r \sqrt{1-\frac{2 v}{E}\left[\frac{\rho_{0} \omega^{2} b^{m} r^{2-m}}{3-m}-\frac{B_{1}}{r}+\frac{\alpha E \Theta_{0}(2-C)}{\ln (a / b)}+\frac{2(2-C) \alpha E \Theta_{0} \ln (r / b)}{\ln (a / b)}\right]}$

where $E=2 \mu(3-2 C) /(2-C)$ is the Young's modulus in term of compressibility factor can be expressed as.

Using boundary condition (3) and (13) in eqs. (19) and (21), one get:

$$
\begin{aligned}
& A=\frac{\rho_{0} \omega^{2} b^{m} n v\left(b^{3-m}-a^{3-m}\right)}{2 \mu(3-m) b^{v}}+\frac{\alpha E \Theta_{0} n(1-C)(b-a)}{2 \mu \ln (a / b) b^{1-C / 2-C}}-\frac{\alpha E \Theta_{0} n a}{\mu b^{1-C / 2-C}} \\
& B=\frac{\rho_{0} \omega^{2} b^{m} a^{3-m}}{(3-m)}+\frac{\alpha E \Theta_{0}(2-C) a}{\ln (a / b)}+\frac{2(2-C) \alpha E \Theta_{0} a}{(1-C)}
\end{aligned}
$$

Substituting eqs. (22) and (23) in eqs. (18), (19), and (21) respectively, one get the transitional stresses and displacement as:

$$
\begin{aligned}
& T_{\theta \theta}=\left(\frac{\rho_{0} \omega^{2} v b^{m}\left(\begin{array}{l}
b^{3-m} \\
-a^{3-m}
\end{array}\right)}{(3-m) r}\left(\frac{r}{b}\right)^{v}+\alpha E \Theta_{0}(2-C)\left[\begin{array}{l}
\frac{\ln (r / b)}{\ln (a / b)}-\frac{2 a}{(2-C) r}\left(\frac{r}{b}\right)^{1-C / 2-C} \\
+\frac{(1-C)(b-a)}{r(2-C) \ln (a / b)}\left(\frac{r}{b}\right)^{1-C / 2-C}
\end{array}\right]\right) \forall \quad m \neq 3 \\
& T_{r r}=\left(\begin{array}{l}
\frac{\rho_{0} \omega^{2} b^{m}}{(3-m) r}\left[\begin{array}{l}
\left(b^{3-m}-a^{3-m}\right)\left(\frac{r}{b}\right)^{v} \\
-r^{3-m}+a^{3-m}
\end{array}\right]+\frac{\alpha E \Theta_{0}(2-C)}{\ln (a / b)}\left[\begin{array}{l}
\ln (r / b)+\frac{a}{r}-1+ \\
\frac{(b-a)}{r}\left(\frac{r}{b}\right)^{1-C / 2-C}
\end{array}\right] \\
+\frac{2 \alpha E \Theta_{0}(2-C)}{(1-C)}\left[\frac{a}{r}-\frac{a}{r}\left(\frac{r}{b}\right)^{1-C / 2-C}\right]
\end{array}\right) \quad \forall \neq 3 \\
& u=r-r \sqrt{1-\frac{2 v}{E}\left\{\begin{array}{l}
\left(\frac{\rho_{0} \omega^{2} b^{m}}{(3-m) r}\left[r^{3-m}-a^{3-m}\right]\right)+\frac{\alpha E \Theta_{0}(2-C)(r-a)}{r \ln (a / b)} \\
+\frac{2(2-C) \alpha E \Theta_{0}}{(1-C)}\left[\frac{\ln (r / b)}{\ln (a / b)}-\frac{a}{r}\right]
\end{array}\right.} \forall \forall m \neq 3
\end{aligned}
$$


$\operatorname{and} T_{r r}-T_{\theta \theta}=\left|\begin{array}{l}\frac{\rho_{0} \omega^{2} b^{2}}{(3-m) R}\left[(1-v)\left(1-R_{0}^{3-m}\right) R^{v}-R^{3-m}+R_{0}^{3-m}\right] \\ +\alpha E \Theta_{0}\left[\begin{array}{l}\frac{2 R_{0}}{R \nu}-\frac{2 R_{0}}{(1-C)} R^{\nu-1}+\left(1-R_{0}\right) R^{\nu-1} \\ +\frac{(2-C)}{\ln R_{0}}\left(\frac{R_{0}-R}{R}\right)\end{array}\right]\end{array}\right| \forall \quad m \neq 3$

where $R_{0}=a / b$ and $R=r / b$ in non dimensional form.

Initial Yielding: The maximum value $\left|T_{r r}-T_{\theta \theta}\right|$ occurs at the radius $R=R_{1}$ (say), which depends upon the value of $m$ and $C$. For example if we take $C=0,0.25,0.5$ yielding starts at the internal surface for $m=-1.9,-1.6,-1.2$ respectively and for values $m=-5.1,-4.9,-3.9$ yielding starts at the mid surface. For the values $m=-3.1 \times 10^{9},-2.9 \times 10^{9},-1.6 \times 10^{9} ;\left|T_{r r}-T_{\theta \theta}\right|$ become neither maximum nor minimum values at the external surface $R_{1}=1$ i.e. yielding does not occurs at the external surface. For yielding at $R=R_{1}$, eq. (27) becomes:

$\left|T_{r r}-T_{\theta \theta}\right|_{R=R_{1}}=\left|\begin{array}{l}\frac{\rho_{0} \omega^{2} b^{2}}{(3-m) R_{1}}\left[(1-v)\left(1-R_{0}^{3-m}\right) R_{1}^{v}-R_{1}^{3-m}+R_{0}^{3-m}\right] \\ +\alpha E \Theta_{0}\left[\frac{2 R_{0}}{R_{1} V}-\frac{2 R_{0}}{(1-C)} R_{1}^{\nu-1}+\left(1-R_{0}\right) R_{1}^{\nu-1}+\frac{(2-C)}{\ln R_{0}}\left(\frac{R_{0}-R_{1}}{R_{1}}\right)\right.\end{array}\right| \equiv Y$ (yielding say)

where $Y$ is the yielding stress.

The angular speed necessary for initial yielding is given by:

$\Omega_{i}^{2}=\frac{\rho_{0} \omega_{i}^{2} b^{2}}{Y}=\left(\left|R_{1} S\right|-\left|\left(\frac{\alpha E \Theta_{0}}{Y}\right) S\left[\frac{2 R_{0}}{v}-\frac{2 R_{0}}{(1-C)} R_{1}^{v}+\left(1-R_{0}\right) R_{1}^{v}+\frac{(2-C)}{\ln R_{0}}\left(\begin{array}{l}R_{0} \\ -R_{1}\end{array}\right)\right]\right|\right)$

where $S=\frac{(3-m) R_{1}}{\left[(1-v)\left(1-R_{0}^{3-m}\right) R_{1}^{v}-R_{1}^{3-m}+R_{0}^{3-m}\right]}$ and $\omega_{i}=\frac{\Omega_{i}}{b} \sqrt{\frac{Y}{\rho_{0}}}$.

We introduce the following non-dimensional components:

$R=r / b, R_{0}=a / b, \sigma_{r}=T_{r r} / Y, \sigma_{\theta}=T_{\theta \theta} / Y, \bar{u}=u / b, \Theta_{1}=\alpha E \Theta_{0} / Y, \Omega^{2}=\rho_{0} \omega^{2} b^{2} / Y$

and $H=Y / E$.

Elastic-plastic transitional stresses, angular speed and displacement from equations (24), (25), (28) and (26) in non-dimensional form become:

$$
\begin{aligned}
& \sigma_{\theta}=\left(\frac{\Omega_{i}^{2} v\left(1-R_{0}^{3-m}\right) R^{\nu-1}}{(3-m)}\right)+\Theta_{1}(2-C)\left[\frac{\left(1-R_{0}\right)(1-C)}{(2-C) \ln R_{0}} R^{\nu-1}+\frac{\ln R}{\ln R_{0}}-\frac{2 R_{0}}{(2-C)} R^{v-1}\right] \\
& \sigma_{r}=\left[\frac{\Omega_{i}^{2}}{R(3-m)}\left[\begin{array}{c}
\left(1-R_{0}^{3-m}\right) R^{v} \\
-R^{3-m}+R_{0}^{3-m}
\end{array}\right]+\frac{\Theta_{1}(2-C)}{\ln R_{0}}\left[\begin{array}{l}
\ln R+\frac{R_{0}}{R}-1 \\
+\left(1-R_{0}\right) R^{v-1}
\end{array}\right]+\frac{2 \Theta_{1}}{v}\left[\frac{R_{0}}{R}\left(1-R^{v}\right)\right]\right.
\end{aligned}
$$


$\Omega_{i}^{2}=\left(\left|R_{1} S\right|-\mid \Theta_{1} S\left[\begin{array}{l}\frac{2 R_{0}}{v}-\frac{2 R_{0}}{(1-C)} R_{1}^{v}+\left(1-R_{0}\right) R_{1}^{v} \\ +\frac{(2-C)}{\ln R_{0}}\left(R_{0}-R_{1}\right)\end{array}\right]\right)$

and $\left.U=R-R \sqrt{1-2 v H\left\{\begin{array}{l}\frac{\Omega_{i}^{2}}{R(3-m)}\left[R^{3-m}-R_{0}^{3-m}\right]+\frac{\Theta_{1}(2-C)\left(R-R_{0}\right)}{R \ln R_{1}} \\ +\frac{2(2-C) \Theta_{1}}{(1-C)}\left[\frac{\ln R}{\ln R_{0}}-\frac{R_{0}}{R}\right]\end{array}\right.}\right\}$

Fully Plastic State: Stresses and displacement at the inner boundary satisfied the inequality $T_{r r}>T_{\theta \theta}>T_{z z}(=0)$ and yielding occurs at the inner radius. For fully-plastic state $C \rightarrow 0$ i.e.v $=1 / 2$. Two plastic zones for fully plastic state were considered as shown in Fig. 2. There are two plastic zones:

(i) Inner-plastic zone:

$T_{r r}>T_{\theta \theta}>T_{z z}(=0) ; a \leq r \leq r_{1}$

or $\sigma_{r}>\sigma_{\theta}>\sigma_{z}(=0) ; R_{0} \leq R \leq R_{1}$.

(ii) Outer-plastic zone:

$T_{\theta \theta}>T_{r r}>T_{z z}(=0), r_{1} \leq r \leq b$

or $\sigma_{\theta}>\sigma_{r}>\sigma_{z}(=0) R_{1} \leq R \leq 1$.

Where $r_{1}$ is the radius of inner plastic zone.

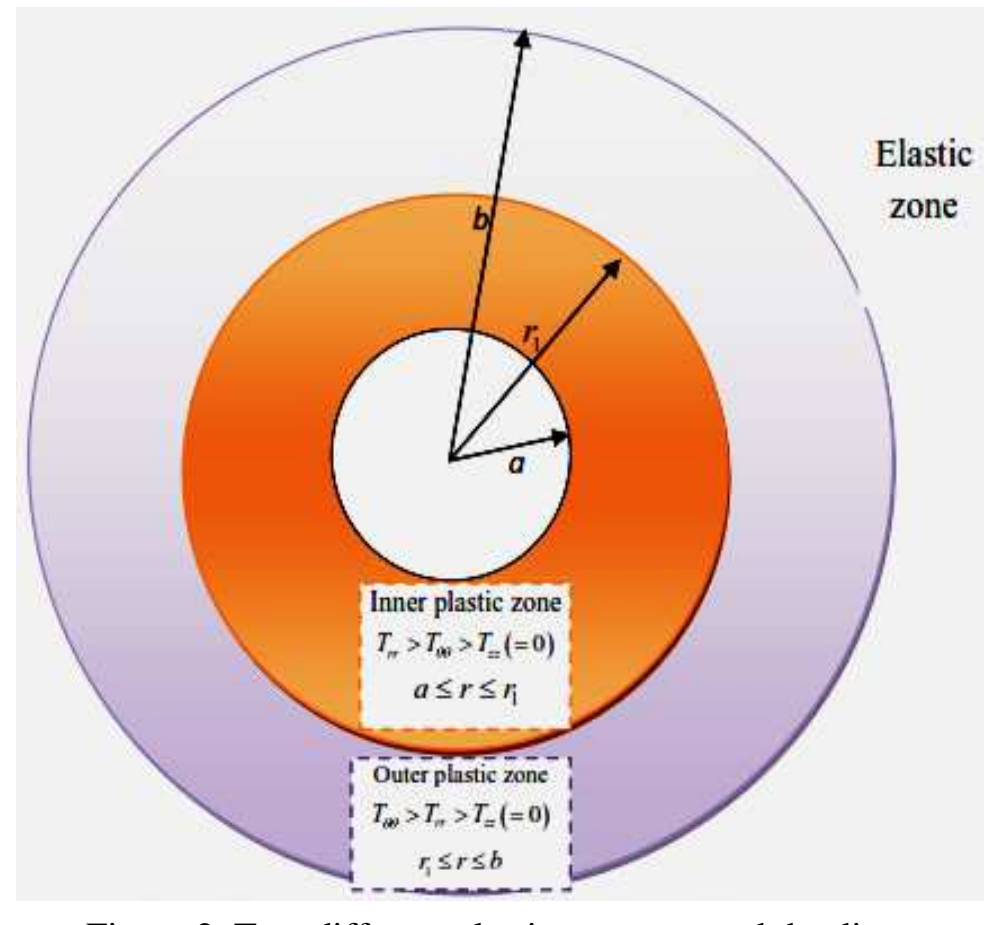

Figure 2. Two different plastic zones around the disc for fully plastic state.

For Inner-plastic zone, eq. (27) becomes:

$\left|T_{r r}-T_{\theta \theta}\right|_{R=R_{0}}=\left|\frac{\rho_{0} \omega_{f^{\prime}}^{2} b^{2}\left(1-R_{0}^{3-m}\right)}{2(3-m) \sqrt{R_{0}}}+\frac{\alpha E \Theta_{0}}{\sqrt{R_{0}}}\left[4 \sqrt{R_{0}}-3 R_{0}+1\right]\right| \equiv Y^{*}($ say $)$

and the angular speed required for fully plastic state is given by:

$\Omega_{f^{*}}^{2}=\frac{\rho_{0} \omega_{f^{*}}^{2} b^{2}}{Y^{*}}=\left[\left|\frac{2(3-m) \sqrt{R_{0}}}{\left(1-R_{0}^{3-m}\right)}\right|-\left|\Theta_{1}\left(\frac{2(3-m)}{\left(1-R_{0}^{3-m}\right)}\right)\left[4 \sqrt{R_{0}}-3 R_{0}+1\right]\right|\right]$

where $\omega_{f^{*}}=\frac{\Omega_{f^{*}}}{b} \sqrt{\frac{Y}{\rho_{0}}}$ and $\frac{\alpha E \Theta_{0}}{Y^{*}}=\Theta_{1}$.

Using equation (33) in eqs. (29), (30), (32) by taking $C \rightarrow 0$ i.e.v $=1 / 2$, we get the stresses and displacement for the inner plastic zone as:

$\sigma_{\theta}^{*}=\left(\frac{\Omega_{f^{*}}^{2}\left(1-R_{0}^{3-m}\right)}{2(3-m) \sqrt{R}}\right)+2 \Theta_{1}\left[\frac{\left(1-R_{0}\right)}{2 \sqrt{R} \ln R_{0}}+\frac{\ln R}{\ln R_{0}}-\frac{R_{0}}{\sqrt{R}}\right]$ 


$$
\begin{aligned}
& \sigma_{r}^{*}=\left[\frac{\Omega_{f^{*}}^{2}}{R(3-m)}\left[\begin{array}{c}
\left(1-R_{0}^{3-m}\right) \sqrt{R} \\
-R^{3-m}+R_{0}^{3-m}
\end{array}\right]+\frac{2 \Theta_{1}}{\ln R_{0}}\left[\ln R+\frac{R_{0}}{R}-1+\frac{\left(1-R_{0}\right)}{\sqrt{R}}\right]+\Theta_{1}\left[\frac{R_{0}}{R}(1-\sqrt{R})\right]\right. \\
& \text { and } U_{f^{*}}=R-R \sqrt{1-H\left\{\frac{\Omega_{f^{*}}^{2}}{R(3-m)}\left[R^{3-m}-R_{0}^{3-m}\right]+\frac{2 \Theta_{1}\left(R-R_{0}\right)}{R \ln \left(R_{0}\right)}+4 \Theta_{1}\left[\frac{\ln R}{\ln R_{0}}-\frac{R_{0}}{R}\right]\right.}
\end{aligned}
$$

For outer plastic zone eq. (27) becomes:

$$
\left|T_{r r}-T_{\theta \theta}\right|_{R=1}=\left|-\frac{\rho_{0} \omega_{f^{*}}^{2} b^{2}\left(1-R_{0}^{3-m}\right)}{2(3-m)}+\alpha E \Theta_{0}\left[R_{0}+1+\frac{2}{\ln R_{0}}\left(R_{0}-1\right)\right]\right|=Y^{* *}(\text { say })
$$

and the and the required angular speed is given by:

$\Omega_{f^{* *}}^{2}=\frac{\rho_{0} \omega_{f^{*}}^{2} b^{2}}{Y^{* *}}=\left|\frac{2(3-m)}{1-R_{0}^{3-m}}\right|-\mid \Theta_{1}\left(\frac{2(3-m)}{1-R_{0}^{3-m}}\right)\left[R_{0}+1+\frac{2}{\ln R_{0}}\left(R_{0}-1\right)\right]$

where $\omega_{f^{* *}}=\frac{\Omega_{f^{* *}}}{b} \sqrt{\frac{Y}{\rho_{0}}}$ and $\frac{\alpha E \Theta_{0}}{Y^{* *}}=\Theta_{1}$.

Using equation (37) in eqs. (29), (30), (32) by taking $C \rightarrow 0$ i.e. $v=1 / 2$, one get the stresses and displacement for the outer plastic zone as:

$$
\begin{aligned}
& \sigma_{\theta}^{* *}=\left(\frac{\Omega_{f^{* *}}^{2}\left(1-R_{0}^{3-m}\right)}{2(3-m) \sqrt{R}}\right)+2 \Theta_{1}\left[\frac{\left(1-R_{0}\right)}{2 \sqrt{R} \ln R_{0}}+\frac{\ln R}{\ln R_{0}}-\frac{R_{0}}{\sqrt{R}}\right] \\
& \sigma_{r}^{* *}=\left[\frac{\Omega_{f^{* *}}^{2}}{R(3-m)}\left[\begin{array}{l}
\left(1-R_{0}^{3-m}\right) \sqrt{R} \\
-R^{3-m}+R_{0}^{3-m}
\end{array}\right]+\frac{2 \Theta_{1}}{\ln R_{0}}\left[\ln R+\frac{R_{0}}{R}-1+\frac{\left(1-R_{0}\right)}{\sqrt{R}}\right]+\Theta_{1}\left[\frac{R_{0}}{R}(1-\sqrt{R})\right]\right] \\
& \text { and } U_{f^{* *}}=R-R \sqrt{1-H\left\{\frac{\Omega_{f^{* *}}^{2}}{R(3-m)}\left[R^{3-m}-R_{0}^{3-m}\right]+\frac{2 \Theta_{1}\left(R-R_{0}\right)}{R \ln R_{0}}+4 \Theta_{1}\left[\frac{\ln R}{\ln R_{0}}-\frac{R_{0}}{R}\right]\right\}}
\end{aligned}
$$

\section{RESULTS AND DISCUSSION}

For calculating the stresses, angular speed and displacement based on the above analysis, the following values have been taken: $C=0.00,0.25,0.5$ and $0.75, \Theta_{0}=700^{\circ} F$ and $\alpha=5.0 \times$ $10^{-5} \operatorname{deg} F^{-1}$ for Methyl Methacrylate [21], $\Theta_{1}=0,0.0175$ and 0.07 respectively.

In Tab. 1, angular speed required for initial yielding $\Omega_{i}^{2}$ and fully-plastic state $\Omega_{f}^{2}$ in a rotating disc having variable density for different values of $m, C$ and $\Theta_{1}$ has been given. It can be seen from the Tab. 1 that yielding occurs at any radius $R=R_{1}$ or at the internal surface $R_{1}=0.5$ or at the mid surface $R_{1}=0.7$ of the disc depending upon the values of $m$ and $C$. For example yielding occurs at the internal surface of the disc made of compressible material $(C=$ 0.25 ) at a angular speed 2.82511676 for $m=-1.6$ whereas yielding occurs at the middle surface at the angular speed 7.72178021 for $m=-3$. It is also seen from tab. 1 that rotating disc having variable density and made of incompressible material yields at a higher angular speed as compare to disc made of compressible material. Compressible material of rotating disc with shaft having variable density required higher percentage increased angular speed to become fully- 
plastic as compare to incompressible material. In Tab. 2, angular speed required for initial yielding $\Omega_{i}^{2}$ and fully-plastic state $\Omega_{f}^{2}$ of a rotating disc for different values of $m=0,2.9$ and $\Theta_{1}=0.00,0.0175,0.07$ has been given. It is seen that with the effect of temperature, rotating disc required higher percentage angular speed to become fully plastic state with increase temperature for $m=2.9$ but reverse in case $m=0$.

Table 1. Angular speed for initial yielding $\Omega_{i}^{2}$ and fully plastic state $\Omega_{f}^{2}$ of a rotating disc for different values of $\mathrm{m}, \mathrm{C}$ and $\Theta_{1}$.

\begin{tabular}{|c|c|c|c|c|c|c|c|c|c|}
\hline & \multirow{2}{*}{$\begin{array}{c}\text { Temperature } \\
\theta_{1}\end{array}$} & \multirow{2}{*}{\begin{tabular}{|c} 
Compressibility \\
C \\
\end{tabular}} & \multirow{2}{*}{\begin{tabular}{|c|}
$\begin{array}{c}\text { Density } \\
\text { variation }\end{array}$ \\
$m$ \\
\end{tabular}} & \multirow{2}{*}{$\begin{array}{l}\text { Yielding } \\
\text { Occurs } \\
\text { at } \\
R\end{array}$} & \multicolumn{2}{|c|}{$\begin{array}{l}\text { Rotating Disc having } \\
\text { Variable density } \\
\rho=\rho_{0}(r / b)^{-m}\end{array}$} & \multirow{2}{*}{\begin{tabular}{|l}
$\begin{array}{l}\text { Angular } \\
\text { speed } \\
\text { required for } \\
\text { initial } \\
\text { yielding }\end{array}$ \\
\multicolumn{1}{|c|}{$\Omega^{2}$} \\
\end{tabular}} & \multirow{2}{*}{$\begin{array}{l}\text { Angular } \\
\text { speed } \\
\text { required for } \\
\text { fully -plastic } \\
\text { state } \\
\\
\qquad \Omega_{y}^{2}\end{array}$} & \multirow{2}{*}{\begin{tabular}{|l}
$\begin{array}{l}\text { Percentage increase in } \\
\text { Angular speed } \\
\%\end{array}$ \\
$\left(\sqrt{\Omega_{j}^{2} / \Omega_{i}^{2}}-1\right) \times 100$
\end{tabular}} \\
\hline & & & & & $r=a$ & $r-b$ & & & \\
\hline \multirow{6}{*}{$0.5 \leq R \leq$} & $\begin{array}{l}0 \\
0.0175 \\
0.07\end{array}$ & $\begin{array}{l}0 \\
0 \\
0\end{array}$ & $\begin{array}{l}-1.9 \\
-1.9 \\
-1.9\end{array}$ & \multirow{3}{*}{$R_{l}=0.5$} & $\begin{array}{l}\rho=\rho_{0}(0.267943) \\
\rho=\rho_{n}(0.267943) \\
\rho=\rho_{0}(0.267943)\end{array}$ & $\begin{array}{l}\rho=\rho_{11} \\
\rho=\rho_{11} \\
\rho=\rho_{11}\end{array}$ & $\begin{array}{l}3.58489172 \\
3.37831007 \\
2.75856513\end{array}$ & $\begin{array}{l}10.13900499 \\
9.617444093 \\
8.050961414\end{array}$ & $\begin{array}{l}68.1792831 \% \\
68.7252503 \% \\
70.8371241 \%\end{array}$ \\
\hline & $\begin{array}{l}0 \\
0.0175 \\
0.07\end{array}$ & $\begin{array}{l}0.25 \\
0.25 \\
0.25\end{array}$ & $\begin{array}{l}-1.6 \\
-1.6 \\
-1.6\end{array}$ & & $\begin{array}{l}\rho=\rho_{0}(0.329877) \\
\rho=\rho_{0}(0.329877) \\
\rho=\rho_{0}(0.329877)\end{array}$ & $\begin{array}{l}\rho=\rho_{0} \\
\rho=\rho_{11} \\
\rho=\rho_{0}\end{array}$ & $\begin{array}{l}2.82511676 \\
2.65562129 \\
2.14713488\end{array}$ & $\begin{array}{l}9.595673992 \\
9.101524002 \\
7.619074033\end{array}$ & $\begin{array}{l}84.2975414 \% \\
85.128801 \% \\
88.3742052 \%\end{array}$ \\
\hline & $\begin{array}{l}0 \\
0.0175 \\
0.07\end{array}$ & $\begin{array}{l}0.5 \\
0.5 \\
0.5\end{array}$ & $\begin{array}{l}-1.2 \\
-1.2 \\
-1.2\end{array}$ & & $\begin{array}{l}\rho=\rho_{0}(0.435275) \\
\rho=\rho_{0}(0.435275) \\
\rho=\rho_{0}(0.435275)\end{array}$ & $\begin{array}{l}\rho=\rho_{11} \\
\rho=\rho_{11} \\
\rho=\rho_{11}\end{array}$ & $\begin{array}{l}2.0985569 \\
1.96565377 \\
1.5669444\end{array}$ & \begin{tabular}{|l|}
8.883337134 \\
8.425870472 \\
7.053470485
\end{tabular} & $\begin{array}{l}105.744246 \% \\
107.039818 \% \\
112.165436 \%\end{array}$ \\
\hline & \begin{tabular}{|l|}
0 \\
0.0175 \\
0.07
\end{tabular} & $\begin{array}{l}0 \\
0 \\
0\end{array}$ & $\begin{array}{l}-3.5 \\
-3.5 \\
-3.5\end{array}$ & \multirow[b]{3}{*}{$R_{1}=0.7$} & $\begin{array}{l}\rho=\rho_{n}(0.286974) \\
\rho=\rho_{0}(0.286974) \\
\rho=\rho_{0}(0.286974)\end{array}$ & $\begin{array}{l}\rho=\rho_{0} \\
\rho=\rho_{0} \\
\rho=\rho_{u}\end{array}$ & $\begin{array}{l}9.76022621 \\
9.23347949 \\
7.05323933\end{array}$ & \begin{tabular}{|l|}
13.14523571 \\
12.46829336 \\
10.43746631
\end{tabular} & $\begin{array}{l}16.0524324 \% \\
16.2039288 \% \\
16.7817277 \%\end{array}$ \\
\hline & $\begin{array}{l}0 \\
0.0175 \\
0.07\end{array}$ & $\begin{array}{l}0.25 \\
0.25 \\
0.25\end{array}$ & $\begin{array}{l}-3 \\
-3 \\
-3\end{array}$ & & $\begin{array}{l}\rho=\rho_{s}(0.343) \\
\rho=\rho_{0}(0.343) \\
\rho=\rho_{0}(0.343)\end{array}$ & $\begin{array}{l}\rho=\rho_{n} \\
\rho=\rho_{i n} \\
\rho=\rho_{n}\end{array}$ & $\begin{array}{l}7.72178021 \\
7.31193343 \\
6.08239309\end{array}$ & $\begin{array}{l}12.19047619 \\
11.56270125 \\
9.679376422\end{array}$ & $\begin{array}{l}25.6468535 \% \\
25.7516053 \% \\
26.1497655 \%\end{array}$ \\
\hline & $\begin{array}{l}0 \\
0.0175 \\
0.07\end{array}$ & $\begin{array}{l}0.5 \\
0.5 \\
0.5\end{array}$ & $\begin{array}{l}-2.5 \\
-2.5 \\
-2.5\end{array}$ & & $\begin{array}{l}\rho=\rho_{0}(0.409963) \\
\rho=\rho_{0}(0.409963) \\
\rho=\rho_{0}(0.409963)\end{array}$ & $\begin{array}{l}\rho=\rho_{n} \\
\rho=\rho_{k} \\
\rho=\rho_{k}\end{array}$ & $\begin{array}{l}5.85442477 \\
5.54692806 \\
4.02443793\end{array}$ & $\begin{array}{l}11.24856042 \\
10.66929147 \\
8.93148461\end{array}$ & $\begin{array}{l}38.6137643 \% \\
38.6888423 \% \\
38.9736135 \%\end{array}$ \\
\hline
\end{tabular}


Table 2. Angular speed required for initial yielding $\Omega_{i}^{2}$ and fully plastic state $\Omega_{f}^{2}$ of a rotating disc for $m=0.00,2.9$ and $\Theta_{1}=0.00,0.0175,0.07$.

\begin{tabular}{|c|c|c|c|c|c|c|c|c|}
\hline & $\begin{array}{c}\text { Temperature } \\
\Theta_{i}\end{array}$ & $\begin{array}{c}\text { Density } \\
\text { variation }\end{array}$ & $\begin{array}{l}\text { Yielding } \\
\text { Occurs at }\end{array}$ & \multicolumn{2}{|c|}{$\begin{array}{l}\text { Rotating Disc having } \\
\text { Variable density } \\
\rho=\rho_{0}(r / b)^{-m}\end{array}$} & $\begin{array}{l}\text { Angular speed } \\
\text { required for initial } \\
\text { yielding }\end{array}$ & $\begin{array}{l}\text { Angular speed } \\
\text { required for fully } \\
\text { plastic state }\end{array}$ & $\begin{array}{l}\text { Percentage increase in } \\
\text { Angular speed }\end{array}$ \\
\hline & & $m$ & $R$ & $r=a$ & $r=b$ & $\Omega_{i}^{2}$ & $\Omega_{f}^{2}$ & $\left(\sqrt{\Omega_{f}^{2} / \Omega_{i}^{2}}-1\right) \times 100$ \\
\hline \multirow[t]{2}{*}{$0.5 \leq R \leq 1$} & $\begin{array}{l}0 \\
0.0175 \\
0.07\end{array}$ & $\begin{array}{l}0 \\
0 \\
0\end{array}$ & $R_{1}=0.5$ & $\begin{array}{l}\rho=\rho_{0} \\
\rho=\rho_{0} \\
\rho=\rho_{0}\end{array}$ & $\begin{array}{l}\rho=\rho_{0} \\
\rho=\rho_{0} \\
\rho=\rho_{0}\end{array}$ & $\begin{array}{l}2.42436611 \\
4.11524145 \\
3.39044371\end{array}$ & $\begin{array}{l}4.8487322 \\
4.569321 \\
3.7310872\end{array}$ & $\begin{array}{l}41.421356 \% \\
5.37271587 \% \\
4.90336764 \%\end{array}$ \\
\hline & $\begin{array}{l}0 \\
0.0175 \\
0.07\end{array}$ & $\begin{array}{l}2.9 \\
2.9 \\
2.9\end{array}$ & $R_{1}=0.7$ & $\begin{array}{l}\rho=\rho_{0}(2.303151) \\
\rho=\rho_{0}(2.303151) \\
\rho=\rho_{0}(2.303151)\end{array}$ & $\begin{array}{l}\rho=\rho_{0} \\
\rho=\rho_{0} \\
\rho=\rho_{0}\end{array}$ & $\begin{array}{l}1.05590319 \\
0.99505611 \\
0.81251484\end{array}$ & $\begin{array}{l}2.986545235 \\
3.024246132 \\
3.137348825\end{array}$ & $\begin{array}{l}68.17928 \% \\
74.33508 \% \\
96.50145 \%\end{array}$ \\
\hline
\end{tabular}

In Figs. 3(a)-3(c), curves have been drawn between stresses and radius ratio $R=r / b$ for fully plastic state at different values of $m=0,2.9,3.5$. It is seen that from figs. 3(a) and 3(c), radial stresses is maximum at the internal surface whereas form fig. 3(b), the circumferential stresses is maximum at the outer surface of the rotating disc. With the introduction of thermal effect it decreases the value of radial and circumferential stresses at inner and outer surface for fully-plastic state.

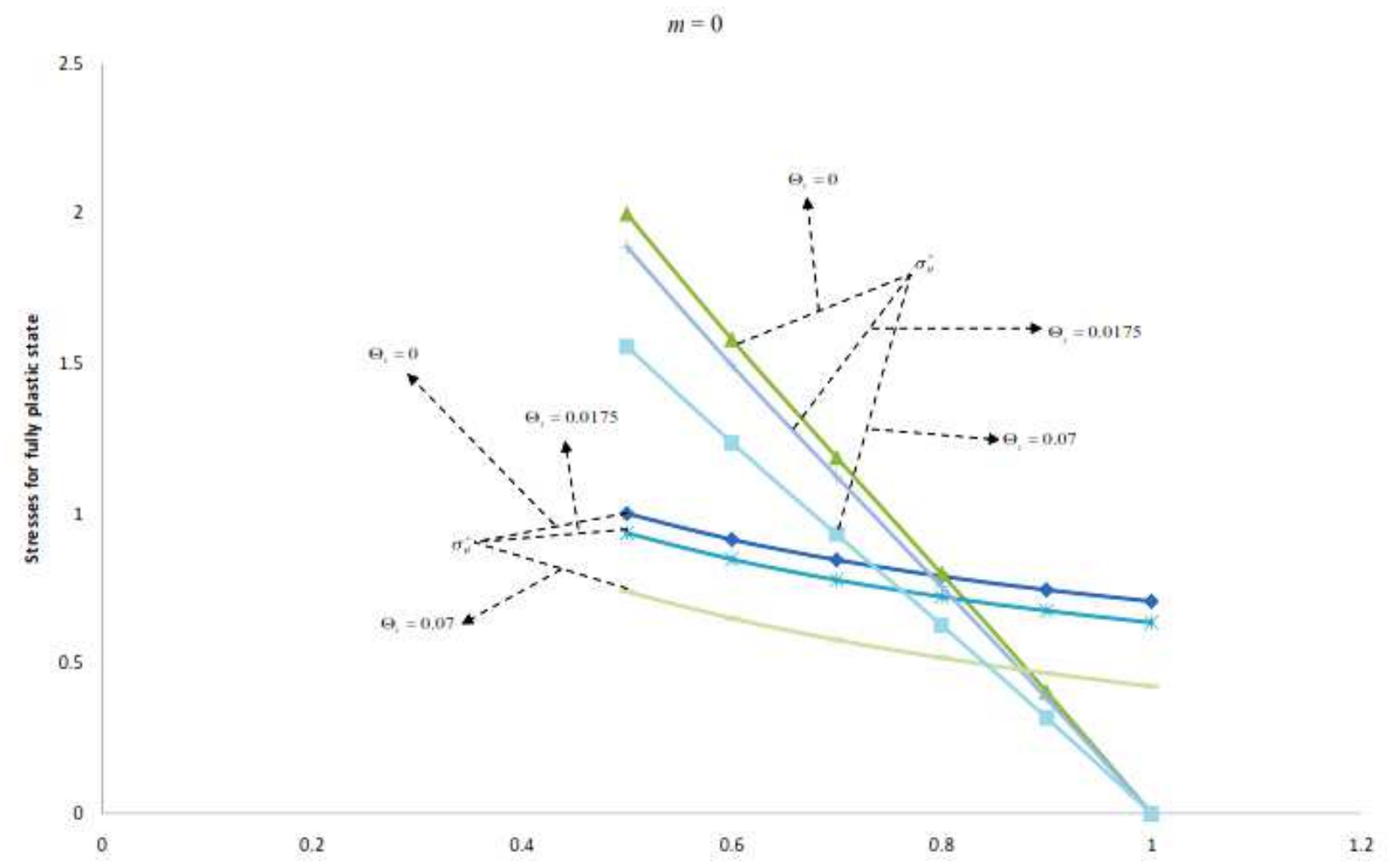

Figure 3(a). Stresses at fully-plastic state for different values of temperature and $m=0.00$ with respect to radii ratio $\mathrm{R}=\mathrm{r} / \mathrm{b}$. 


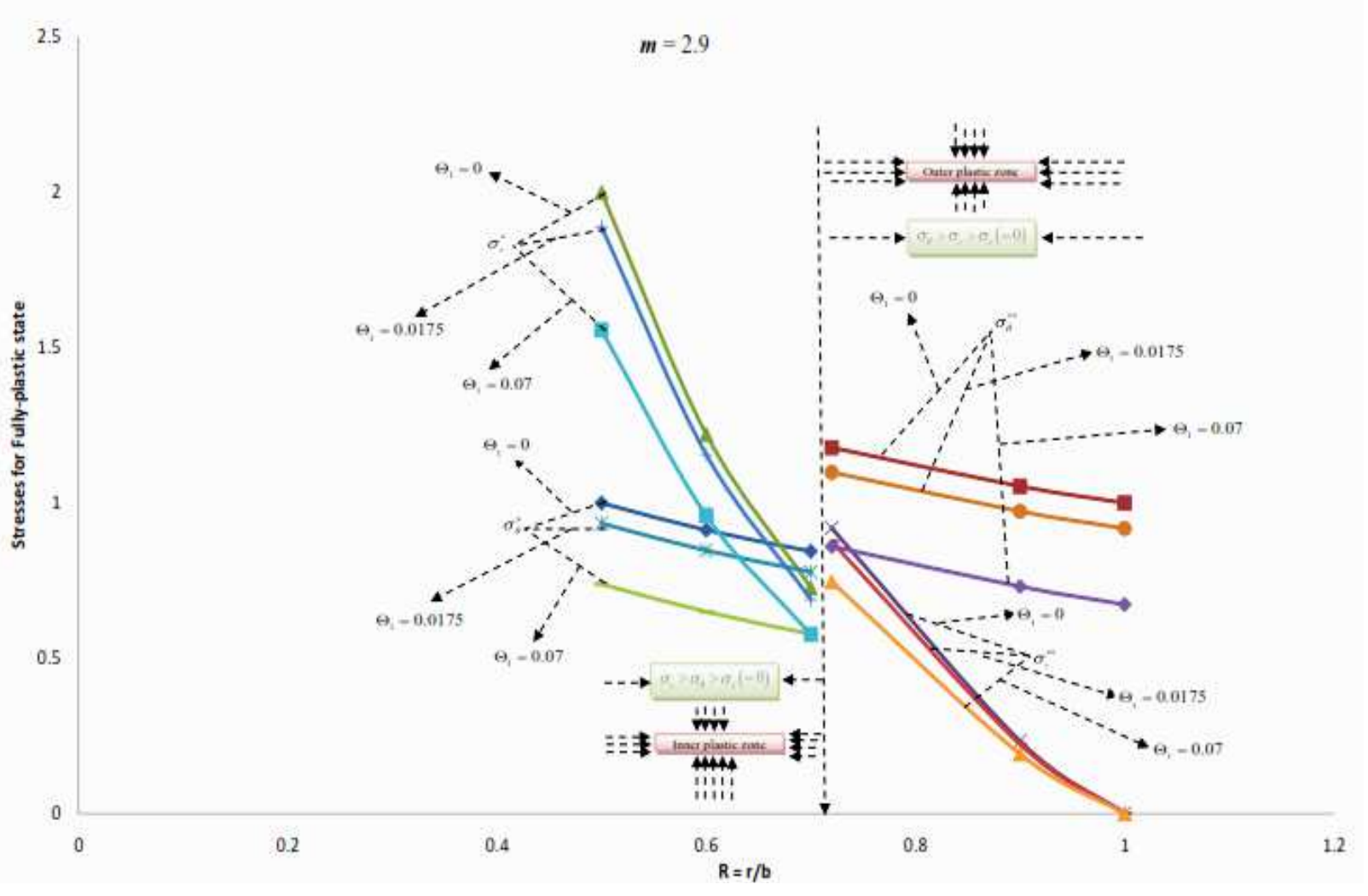

Figure 3(b). Stresses at fully-plastic state for different values of temperature and $m=2.9$ with respect to radii ratio $\mathrm{R}=\mathrm{r} / \mathrm{b}$.

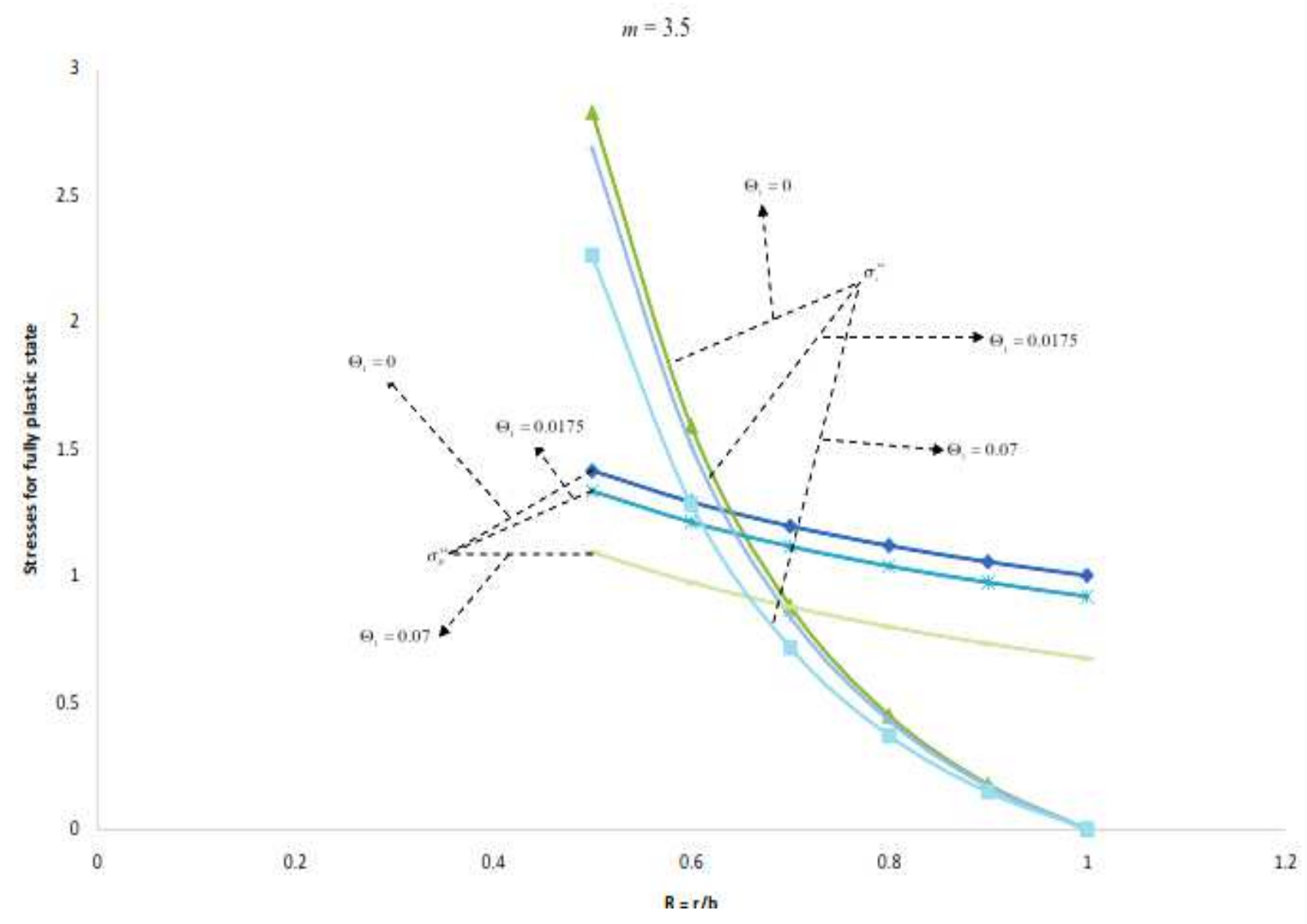

Figure 3(c). Stresses at fully-plastic state for different values of temperature and $m=3.5$ with respect to radii ratio $\mathrm{R}=\mathrm{r} / \mathrm{b}$. 


\section{CONCLUSION}

It has been seen that compressible material required higher percentage increased angular speed to become fully-plastic as compare to rotating disc made of incompressible material. Compressible material of rotating disc with shaft having variable density required higher percentage increased angular speed to become fully-plastic as compare to incompressible material. Circumferential stresses are maximal at the outer surface of the rotating disc. With effect of thermal load value of radial and circumferential stresses at inner and outer surface for fully-plastic state must be decrease.

\section{Acknowledgement}

The author wishes to acknowledge his sincere thanks to Respected Prof. Simeon Oka (Editor-in-Chief of Thermal Science) and Dr Vukman Bakić (Editor of Thermal science) for his encouragement during the preparation of this paper.

\section{Nomenclature}

$a, b \quad$ - Inner and outer radii of the disc $[\mathrm{m}]$,

$\omega \quad$ - Angular velocity of rotation, $\left[s^{-1}\right]$

$u, v, w$ - Displacement components, $[\mathrm{m}]$

$\rho \quad$ - Density of material, $\left[\mathrm{kgm}^{-3}\right]$

$C$ - Compressibility, [ - ]

$T_{i j}, e_{i j}$-Stress $\left[\mathrm{kgm}^{-1} \mathrm{~s}^{-2}\right]$ and Strain rate tensor

$Y \quad$ - Yield stress, $\left[\mathrm{kgm}^{-1} \mathrm{~s}^{-2}\right]$

\section{Greek letters}

$R=r / b ; R_{0}=a / b$ Radii ratio, [-]

$\sigma_{r}$ - Radial stress component $\left(T_{r r} / Y\right),[-]$

$\sigma_{\theta}$ - Circumferential stress component $\left(T_{\theta \theta} / Y\right),[-]$

$\Theta \quad$ - Temperature, $\left[{ }^{0} \mathrm{~F}\right]$

$A, B, d$ - Constants of integration, [ - ]

\section{References:}

[1]Chakrabarty, J., Applied plasticity, Springer-Verlag, New York/Berlin/Heidelberg, 2000.

[2] Heyman, J., Plastic design of rotating discs, Proc. Inst. Mech. Engrs. 172 (1958) 531-546.

[3] Hetnarksi, R.B., IGNACZAK, J., Mathematical Theory of Elasticity, Taylor and Francis, New York, USA, 2004, pp. 60.

[4]SETH, B.R., Transition theory of elastic-plastic deformation, creep and relaxation, Nature, 195 (1962) 896-897. 
[5] SETH, B.R., Measure concept in mechanics, Int. J. Non-linear Mech. I (1966) 2, 35-40.

[6] PANKAJ, T., Some problems in elastic-plastic and creep transition, Ph.D. Thesis, Department of Mathematics, H.P.U. Shimla, India, 2006.

[7] GuPTA, S.K., and PANKaJ, T., Creep transition in a thin rotating disc with rigid inclusion, Defence Science Journal, India 57 (2007) 2, 185-195.

[8] PANKAJ, T., and GUPTA S.K., Thermo elastic-plastic transition in a thin rotating disc with inclusion, Thermal Science 11 (2007) 1, 103-118.

[9] PANKAJ, T., and GuPTA, S.K., Creep transition in an isotropic disc having variable thickness subjected to internal pressure, Proceeding National Academy of Science, India, Section A 78 (2008) I, 57-66.

[10] PANKAJ, T., Elastic-plastic transition stresses in a transversely isotropic thick-walled cylinder subjected to internal pressure and steady state temperature, Thermal Science $\mathbf{1 3}$ (2009) 4, 107-118.

[11] PANKAJ, T., Elastic-plastic transition stresses in a thin rotating disc with rigid inclusion by infinitesimal deformation under steady state temperature, Thermal Science 14 (2010) 1, 209219.

[12] PANKAJ, T., Creep transition stresses in a thin rotating disc with shaft by finite deformation under steady state temperature, Thermal Science 14 (2010) 2, 425-436.

[13] PANKAJ, T., Elastic-plastic transition stresses in rotating cylinder by finite deformation under Steady-State temperature, Thermal Science 15 (2011) 2, 537-543.

[14] PankaJ, T., Creep transition stresses of a thick isotropic spherical shell by finitesimal deformation under steady state of temperature and internal pressure, Thermal Science $\mathbf{1 5}$ (2011), Suppl. 2, pp. S157-S165.

[15] PANKaJ, T., Elastic-plastic transitional stresses in a thin rotating disc with loading edge, Proceeding of International conference on Advances in Modeling, optimization and Computing (AMOC-2011), Department of Mathematics, Indian Institute of Technology Roorkee, Roorkee, Dec. 5-7 (2011) 318-326.

[16] PANKAJ, T., Creep transition stresses in a spherical shell under internal pressure by using lebesgue measure concept, International journal Applied Mechanics and Engineering, Poland 16 (2011) 3, 83-87.

[17] PankaJ, T., Steady thermal stress and strain rates in a circular cylinder with nonhomogeneous compressibility subjected to thermal load, Thermal Science (2012) online: DOI Reference: 10.2298/TSCI110318079P.

[18] PANKAJ, T., Steady thermal stress and strain rates in a rotating circular cylinder under steady state temperature, Thermal Science (2012) online: DOI Reference: 10.2298/TSCI110315080P.

[19] PANKAj, T., Stresses in a thin rotating disc of variable thickness with rigid shaft, Journal for Technology of Plasticity 37 (2012) 1, 1-14.

[20] PARKus, H., Thermo-Elasticity, Springer-Verlag Wien, New York, USA (1976) pp. 30.

[21] LeVITSKy, M. and SHAFFer, B.W., Residual thermal stresses in a solid sphere form a thermosetting material, Jr. of Appl. Mech., Trans. of ASME 42 (1975) 3, 651-655. 